\title{
Inhibition of glutathione-S-transferase as a treatment strategy for multidrug resistance in childhood rhabdomyosarcoma
}

\author{
GUIDO SEITZ ${ }^{1 *}$, MICHAEL BONIN ${ }^{2 *}$, JÖRG FUCHS ${ }^{1}$, SVEN POTHS $^{2}$, PETER RUCK $^{3}$, \\ STEVEN W. WARMANN ${ }^{1}$ and SORIN ARMEANU-EBINGER ${ }^{1}$ \\ ${ }^{1}$ Department of Pediatric Surgery, University Children's Hospital, Hoppe-Seyler-Strasse 3, 72076 Tübingen; \\ ${ }^{2}$ Institute of Anthropology and Human Genetics, Microarray Facility, University Hospital, Calwer Strasse 7, \\ 72076 Tübingen; ${ }^{3}$ Institute of Pathology, Rutesheimer Strasse 50/1, 71229 Leonberg, Germany
}

Received July 20, 2009; Accepted September 8, 2009

DOI: 10.3892/ijo_00000523

\begin{abstract}
Multidrug resistance (MDR) is a common problem in the treatment of childhood rhabdomyosarcoma (RMS). A complete reversal of MDR is currently not possible. The aim of this study was to investigate the role of glutathione-Stransferase (GST) as mechanism of MDR in childhood RMS and to analyze possible reversal strategies. Female athymic mice underwent xenotransplantation with embryonal or alveolar RMS cells and were treated with vincristine. Gene expression analysis using Affymetrix HU-Gene 1.0 arrays revealed 2314 differentially expressed genes between the groups in alveolar RMS and 1387 in embryonal RMS. Ingenuity pathway analysis revealed a cluster of 5 overexpressed genes of the GST family in animals treated with vincristine, putative mediating the development of MDR. In order to analyze possible GST activity after chemotherapy with other commonly used drugs (doxorubicin, topotecan), cell culture experiments with alveolar and embryonal RMS cells were carried out. Specific GST activity was quantified using the clorodinitrobenzol conjugation with glutathione. Increased GST activity was found after incubation with cytotoxic agents in all cell lines. Highest induction of GST activity was found in embryonal RMS (up to 12-fold). After incubation with the GST inhibitors, tumor cell viability was decreased depending on the type of tumor cell and inhibitor used. We detected a novel mechanism for MDR in childhood RMS mediated via genes and proteins of the GST family. Reversal of these effects may be achieved by GST inhibitors
\end{abstract}

Correspondence to: Dr Guido Seitz, Department of Pediatric Surgery, University Children's Hospital, Hoppe-Seyler-Strasse 1, 72076 Tübingen, Germany

E-mail: guido.seitz@med.uni-tuebingen.de

*Contributed equally

Key words: glutathione-S-transferase, rhabdomyosarcoma, multidrug resistance in part. The GST family represents a promising target for further treatment strategies in childhood RMS.

\section{Introduction}

Rhabdomyosarcoma (RMS) is the most common pediatric soft tissue sarcoma. About two-thirds of all sarcomas and $7-8 \%$ of all solid malignant tumors in childhood are RMS (1). The two main histological subtypes are embryonal (eRMS) and alveolar (aRMS) RMS (2). The prognosis of the patients depends on the primary tumor localization $(2,3)$, histological subtype (4), stage of disease (5) and the age at diagnosis (2).

Treatment of childhood RMS is mostly performed within clinical trials such as the Cooperative Soft Tissue Sarcoma Study (CWS 2002-P (6)), SIOP's (International Society of Pediatric Oncology) MTT (Malignant Mesenchymal tumors) trial (7), trials of the Children's Oncology Group (COG 8), or the Italian Soft Tissue Sarcoma Cooperative Group (9). Besides local tumor control with radiotherapy and/or surgery, systemic therapy with cytotoxic agents is important for a sufficient therapy of these patients. The most commonly used cytotoxic agents include vinca-alkaloids, actinomycin D, alkylating agents and antracyclines (6-9).

One major treatment problem, besides local tumor recurrence and development of metastases, is the development of multidrug resistance (MDR), which leads to insufficient response, especially in patients with advanced or relapsed tumors. Various resistance-associated genes and proteins have been identified in RMS (10). We have previously demonstrated that MDR mechanisms in RMS depend on the histological subtype. We found that MDR is mediated via a P-glycoprotein-dependent mechanism in aRMS in vitro and in vivo. In eRMS, mechanisms like multidrug resistanceassociated protein (MRP) or lung resistance-related protein (LRP) seems to play a role for multidrug resistance in vitro. In vivo, these xenobiotic pumps were of less importance (10). Therefore, MDR in childhood RMS is not completely understood and other mechanisms may also play a role in these patients.

Detoxification is another common mechanism for MDR. Gluthatione-S-transferases (GST) belongs to a family of 
Phase II detoxification enzymes that catalyze the conjugation of glutathione (GSH) to a variety of endogenous and exogenous electrophilic components (11). GSTs have been suspected to play a role in the development of drug resistance against cytotoxic agents (11). GSTs might mediate the development of MDR via direct detoxification and as an inhibitor of the MAP kinase pathway. Up-regulation of GSTs has been reported in several cancer types (12) including melanoma cells which were resistant to vincristine (13). Moreover, a reversal of these effects was also seen after the usage of GST inhibitors like dicumarol or curcumin (13). Therefore, a broad range of GST inhibitors were developed to modulate drug resistance and to sensitize tumor cells to cytotoxic drugs $(11,14)$. The role of GSTs for MDR in childhood rhabdomyosarcoma has not been analyzed yet.

The aim of this study was to evaluate the possible role of GST as mediator for MDR in childhood rhabdomyosarcoma. Additionally, a possible reversal of GST by GST inhibitors is discussed.

\section{Material and methods}

Animals and xenotransplantation. Female athymic (nu/nu) NMRI mice, aged 6-8 weeks, weighing 20-25 g, were used in all experiments ( $n=3$ per group). The animals were obtained from our own facility. The animals were kept under pathogen free conditions, fed an autoclaved standard diet and given free access to sterilized water. All animal studies were carried out under principles of laboratory animal care and were approved by the local government ethics committee for animal studies (Tübingen, Germany, CK01/03).

The eRMS cell line A204 (ATCC, Manassas, VA, USA) and the aRMS cell line Rh30 (DSMZ, Braunschweig, Germany) were used for the experiments and were cultured in DMEM medium supplemented with $10 \%$ fetal calf serum, 4.5\% L-Glu and 2.5\% HEPES in a humified atmosphere containing $5 \% \mathrm{CO}_{2}$ at $37^{\circ} \mathrm{C}$. All cell cultures were mycoplasma species negative. Tumor cells were trypsinized and resuspended in RPMI-1640 medium (Gibco, Berlin, Germany). Approximately $10^{6}$ tumor cells $(2 \mathrm{ml})$ were injected subcutaneously in the right flank of nude mice (NMRI nu/nu). Cells from both cell lines were xenotransplanted into 3 mice each. The first group of xenotransplantated animals was not treated with cytotoxic drugs and served as a control group. The second group of animals was treated with the commercially available drug vincristine (VincristinsulfatGRY 5, GRY-Pharma, Kirchzarten, Germany, 0.75 mg/kg day i.p.) as single agent on day 1 and 2 according to the treatment protocol of the Cooperative Soft Tissue Sarcoma Study (CWS 2002-P) of the German Society of Pediatric Hematology and Oncology (GPOH). Vincristine was chosen due to its strong antitumor effects as well as induction of multidrug resistance as previously shown (10). It was given in equitoxic doses for mice. Transplanted animals were continuously observed and clinically examined. After reaching a tumor volume of $0.5 \mathrm{~cm}^{3}$, tumors were resected under general anesthesia and animals were sacrified.

Histological analysis of xenograft tissue specimen. Tumor specimen were fixed in formalin $(37 \%)$ and processed for histological analysis. Tissue processing was continued in a vacuum tissue processor (Leica TP 1050, Leica Wiesloch, Germany). Tissue was paraffin-embedded after processing. Sections $(5 \mu \mathrm{m})$ were made and tissue was deparaffinized with xylole and ethanol. Staining with hematoxylin and eosin was carried out for evaluation of histological changes, mitotic changes and necrosis rate at 10 high power fields (x400).

RNA extraction and linear amplification. Tumor specimens were immediately fixed in liquid nitrogen and stored at $-80^{\circ} \mathrm{C}$ for further gene chip analysis. Therefore, total RNA extraction was carried out using RNeasy Kit (Qiagen, Hilden, Germany). The quality of total RNA of tumors was monitored by Agilent 2100 Bioanalyzer using the RNA 6000 Nano LabChip Kit (Agilent Technologies, Böblingen, Germany) as specified by the manufacturer.

Oligonucleotide microarrays and acquisition of data. For gene chip analysis, tumor specimens from 3 animals of each group were randomly selected. Affymetrix high-density oligonucleotide microarrays (GeneChip HU Gene 1.0, Affymetrix, Santa Clara, CA, USA) were used for gene expression analysis. Hybridization experiments and evaluation was done by the Microarray Facility Tübingen. Arrays were scanned using the GCS3000 Gene Chip scanner (Affymetrix) and GCOS 1.4 software. Scanned images were subjected to visual inspection to control for hybridization artifacts and proper grid alignment and analyzed with Expression Console 1.0 (Affymetrix) to generate report files for quality control. For statistical data analysis the CEL-files from the HG-U133 $2.0+$ arrays were imported into Genespring 7.3 (Agilent Technologies) using Genespring's implementation of GCRMA for normalization and probe summarization (15). Genes that showed an at least two-fold increase or decrease in average expression were analyzed in a Welsh's t-test for significant differences and corrected for multiple testing according to Benjamini and Hochberg (16). In the samples the mRNA of a gene was considered expressed ('present') when the detection $\mathrm{p}$-value and change p-value were $<0.05$. The same was valid for Rh30 and A204 tumors changed in expression during treatment with vincristine. To determine the p-values, a signed rank analysis was carried out on the PM (present match) and MM (mismatch) differences comparing each probe pair. The resulting $p$-values were used to make the change calls. Genes with significantly varied expression in vincristine-treated versus untreated tumors were identified using Data Mining Tool (Affymetrix). Biological mechanisms, pathways and functions of the selected genes were identified by ingenuity pathways analysis. Raw data are available on request from the authors.

Cell culture experiments for GST experiments. After identification of target genes within the GST family, cell culture experiments were performed using the embryonal rhabdomyosarcoma cell lines A204 as well as the alveolar rhabdomyosarcoma cell line Rh30. These cells were cultured as described above. At day one, embryonal and alveolar rhabdomyosarcoma cells $\left(5 \times 10^{4}\right.$ cells $)$ were seeded in 24 -well plates (Becton-Dickinson Falcon Labware, Franklin Lakes, NJ, USA) and were cultured as described above. At day 

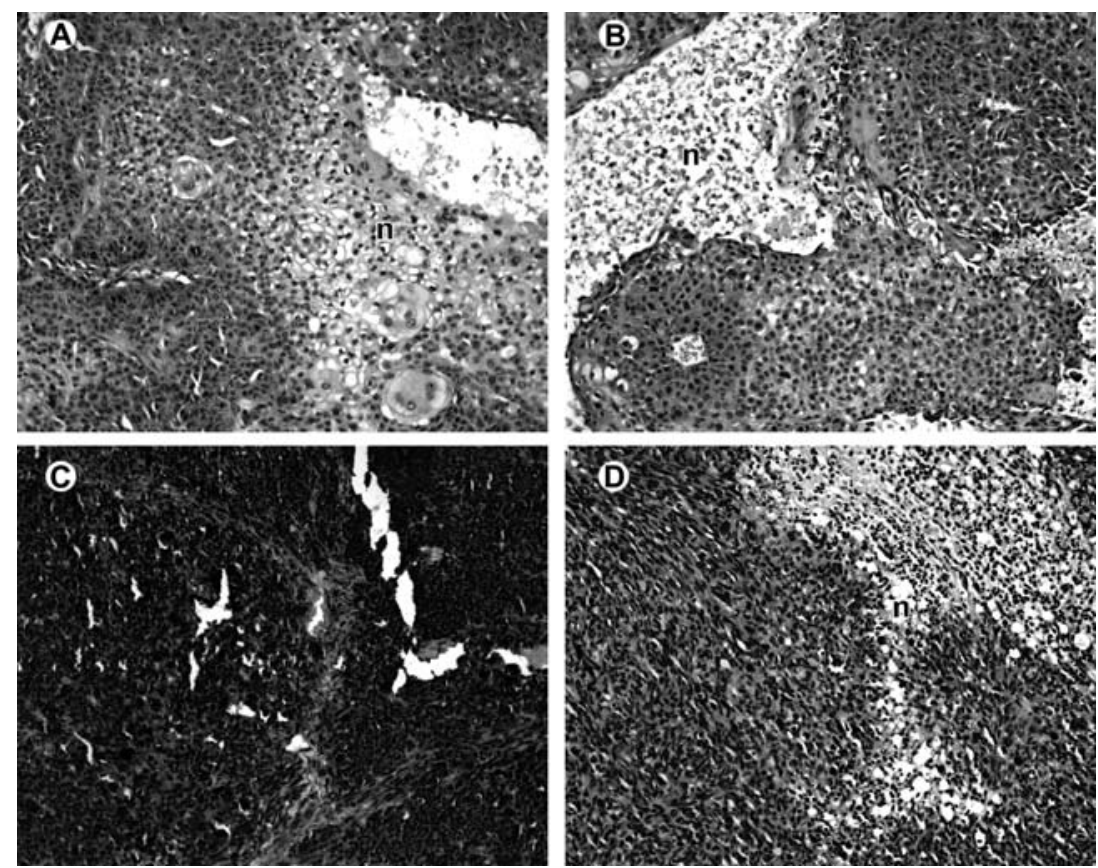

Figure 1. Histological findings in xenotransplants of RMS. Embryonal rhabdomyosarcoma cells A204 (A and B) and alveolar rhabdomyosarcoma Rh30 $(\mathrm{C}$ and $\mathrm{D})$ were treated with vincristine (B and D) or used as controls (A and C). H\&E staining revealed tumor cells of embryonal (A and B) type, alveolar histology (C and D), and necrotic areas (N). Vital tumor cells can still be detected after chemotherapy.

two, commercially available cytotoxic agents vincristine (Vincristinsulfat-GRY5, GRY-Pharma, Kirchzarten, Germany, $1 \mathrm{ng} / \mathrm{ml})$, doxorubicin $(100 \mathrm{ng} / \mathrm{ml})$ and topotecan (Hycamptin, Glaxo Smith Kline, Munich, Germany, $100 \mathrm{ng} / \mathrm{ml}$ ) were added to the cells at $\mathrm{IC}_{50}$ concentrations, which were determined prior to the experiments using the MTT [3-(4,5dimethylthiazol-2-yl)-2,5-diphenyltetrazolium bromide]assay (Biomedica EZ4U, Biozol, Eching, Germany). Drugs were prepared immediately before administration. In the first experiment, cells were incubated for $72 \mathrm{~h}$ with cytotoxic agents. In the second experiment, cells were incubated for $144 \mathrm{~h}$. Cytotoxic agents were renewed after $72 \mathrm{~h}$. These two time points were chosen in order to early evaluate acute effects as well as effects after a longer incubation period. Afterwards, cells were washed with phosphate buffer without $\mathrm{Ca}^{2+}$ and $\mathrm{Mg}^{2+}$ (PBS) and stored in PBS at $-20^{\circ} \mathrm{C}$ until performing the GST assay. All assays were performed 3 times in quadruplicates.

GST assay. A broad range of GST isozymes conjugate the thiol group of glutathione to the substrate 1-chloro-2,4dinitrobenzene (CDNB). Therefore, cells were homogenized in PBS by ultrasonic disruption for 5 pulses at $50 \%$ power (20 kHz Sonifier S-250, Branson Ultrasonic Corporation, Danbury, CT, USA). Total protein concentration was determined by RC DC Protein Assay (BioRad, Munich, Germany). Aliquots of cell homogenates were mixed with $1 \mathrm{mM}$ CDNB and $2 \mathrm{mM}$ L-glutathione in PBS and conjugation of substrate was monitored at $340 \mathrm{~nm}$ and $37^{\circ} \mathrm{C}$ with the ELISA reader (Tecan Spectra Mini, Grödig, Austria). The change in absorbance was determined by plotting the absorbance values against time. Specific GST activity was calculated dividing the absorbance change per minute with the extinction coefficient for CDNB ( $\varepsilon=5.3 \mathrm{mM}-1)$ and the total protein content of the cell homogenate. An induction of the GST activity was assumed if the fold change was greater than 1 (compared to untreated cells).

Inhibition of GST activity. For experiments evaluating a possible inhibition of GST activity by the GST inhibitors OZO-H (4-phenyl-1,3,2-oxathiazolylium-5-oleate) or etacrinic acid (E4754, Sigma-Aldrich, Munich, Germany), cells were prepared as described above. Cells were then incubated with cytotoxic agents (vincristine, doxorubicin and topotecan) in different concentrations (vincristine: 0.033 , $0.14,0.25$ and $1 \mathrm{ng} / \mathrm{ml}$; doxorubicin $0.033,0.14,0.25$ and $1 \mu \mathrm{g} / \mathrm{ml}$; topotecan: $0.033,0.14,0.25$ and $1 \mu \mathrm{g} / \mathrm{ml}$ ) for $72 \mathrm{~h}$ as described above. In order to avoid unspecific cell death, we maintained concentrations of the solvent DMSO lower than $0.1 \%$. In one group, no additional treatment was carried out. This group served as control group. In the other group, cells were additionally incubated with different concentrations of the GST inhibitors etacrinic acid (5, 10, 20 and $40 \mu \mathrm{M})$ or OZO-H (12.5, 25, 50 and $100 \mu \mathrm{M}$; Cayman Chemical, Ann Arbor, MI, USA) for the same time period. Afterwards, cells were washed with PBS. Cell vitality was assessed by the MTT assay.

Statistical analysis. Statistical analysis between the groups was carried out using one way ANOVA on ranks test using GraphPad Prism 4.00 (GraphPad Software, La Jolla, CA). All numeric data are expressed as mean \pm SD. Significance was assumed for all results at $\mathrm{p}<0.05$.

\section{Results}

Histology of xenotransplanted RMS. Standard histology revealed a highly malignant RMS of embryonal (A204) and 


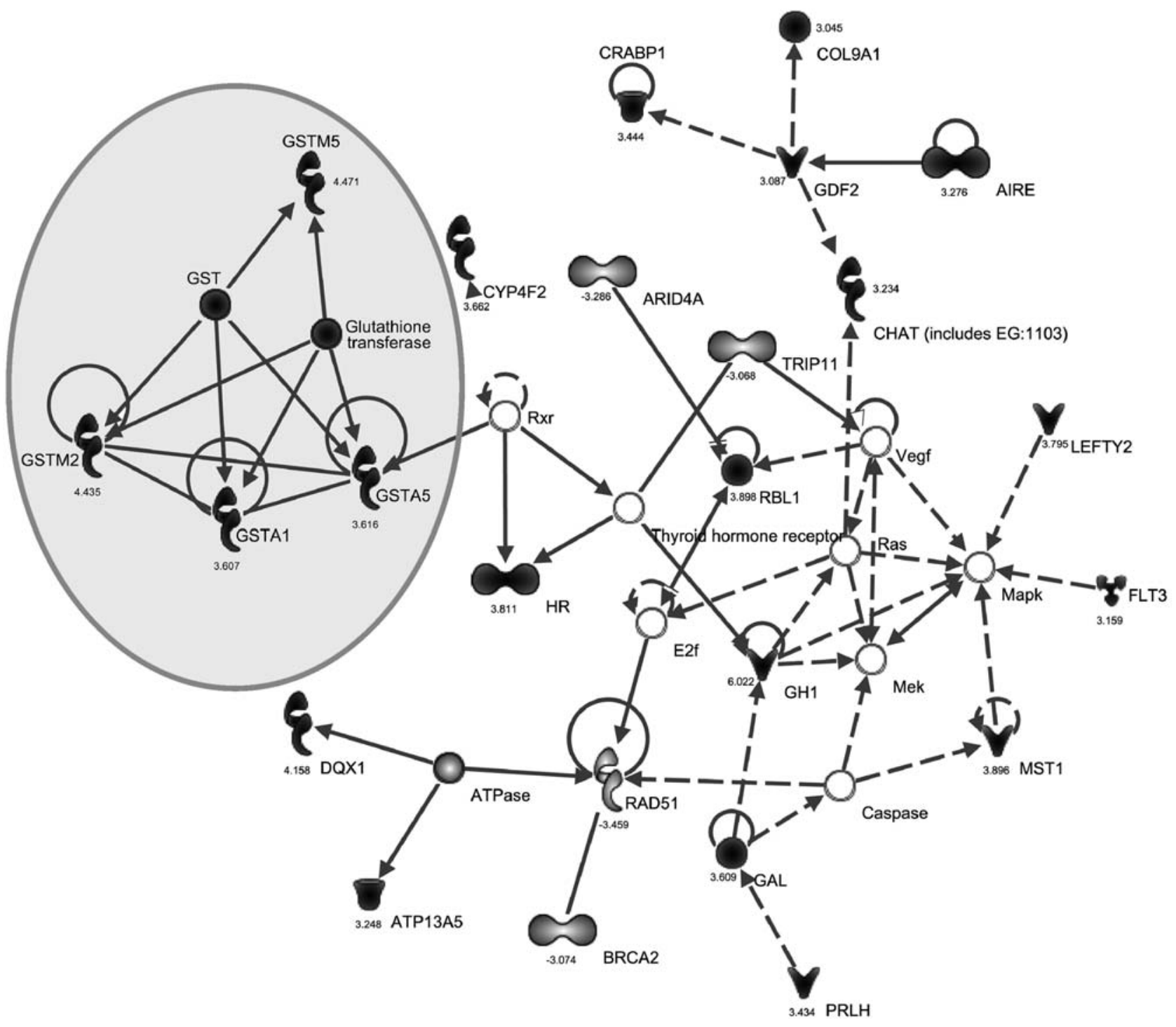

Figure 2. Pathway analysis of differentially expressed genes in alveolar and embryonal RMS after treatment with vincristine. Black staining represents more than 2-fold induction of gene expression by vincristine, grey staining depicts genes 2-fold down-regulated upon treatment with vincristine. The numbers under the symbol represent the mean fold change. Members of the glutathione-S-transferases are highlighted in the grey circle.

alveolar (Rh30) subtype (Fig. 1). The proliferation index expressed as the number of mitotic cells per 10 high power fields ranged between 6 and 45 for A204 tumors and was between 66 and 127 for Rh30 tumors. Tumor tissue revealed necrotic areas and apoptotic bodies over 10 to $30 \%$ of the specimen. There was no difference of the proliferation index and necrotic areas between controls and vincristine-treated tumors. Despite the repeated treatment with vincristine, vital tumor cells were still detected by histological analysis. These cells were considered as resistant toward vincristine.

Influence of vincristine on gene expression in RMS. Gene expression analysis revealed 2314 differentially (SLR >2; SLR: signal log ratio) expressed genes between the groups in alveolar RMS and 1387 differentially (SLR>2) regulated genes in embryonal RMS. We then searched for genes, which were commonly regulated after vincristine treatment in aRMS and eRMS, and were associated with multidrug resistance (MDR). We found genes regulating ATP transporters such as
ATP-binding cassette (ABC1), cadherin 17, liver intestine cadherin, double C2-like domains (B), RAB interacting factor, as well as solute carrier family 25 , synaptic vesicle glycoprotein 2B, as well as MDR/TAP. Pathway analysis clustered 5 members of the GST network (GST, GSTA1, GSTA5, GSTM2 and GSTM5) with increased expression in animals treated with vincristine. The regulations of genes of the GST family as well as common pathways are shown in Fig. 2. Among the entire list of GST isoforms (Table I), GST- $\pi$ was highly overexpressed in all tumor samples. A major interaction molecule in the function of GST- $\pi$, the Jun kinase JNK1, was detected at high level in alveolar RMS and was down-regulated by vincristine to 2.3 -fold. In embryonal RMS, the expression of JNK1 was 4 times lower than in alveolar RMS and was not changed by vincristine. Ingenuity pathway analysis revealed additionally induction of gene expression involved in mechanisms of DNA repair. A list of the 10 most prominent molecule networks detected by pathway analysis are listed in Table II. The given score indicates the 
Table I. Expression of GST isoforms in childhood rhabdomyosarcoma.

\begin{tabular}{|c|c|c|c|c|c|c|c|c|}
\hline $\begin{array}{l}\text { Transcript } \\
\text { cluster ID }\end{array}$ & Gene title & Gene symbol & $\begin{array}{c}\text { Rh30 } \\
\text { (mean } \\
\text { log signal) }\end{array}$ & $\begin{array}{c}\text { Rh30 + } \\
\text { vincristine } \\
\text { (mean log } \\
\text { signal) }\end{array}$ & $\begin{array}{c}\text { RH30 } \\
\text { fold } \\
\text { change }\end{array}$ & $\begin{array}{c}\text { A204 } \\
\text { (mean log } \\
\text { signal) }\end{array}$ & $\begin{array}{c}\text { A204+ } \\
\text { vincristine } \\
\text { (mean log } \\
\text { signal) }\end{array}$ & $\begin{array}{c}\text { A204 } \\
\text { fold } \\
\text { change }\end{array}$ \\
\hline 8127072 & $\begin{array}{l}\text { glutathione S- } \\
\text { transferase A1 }\end{array}$ & GSTA1 & 3.9 & 5.7 & 3.6 & 4.1 & 3.9 & 0.8 \\
\hline 8127065 & $\begin{array}{l}\text { glutathione S- } \\
\text { transferase A2 }\end{array}$ & GSTA2 & 2.8 & 4.1 & 2.3 & 3.5 & 3.4 & 0.9 \\
\hline 8127087 & $\begin{array}{l}\text { glutathione S- } \\
\text { transferase A3 }\end{array}$ & GSTA3 & 3.7 & 3.6 & 1.0 & 3.8 & 3.8 & 1.0 \\
\hline 8127094 & $\begin{array}{l}\text { glutathione S- } \\
\text { transferase A4 }\end{array}$ & GSTA4 & 7.1 & 6.5 & 0.7 & 6.8 & 7.0 & 1.2 \\
\hline 8127079 & $\begin{array}{l}\text { glutathione S- } \\
\text { transferase A5 }\end{array}$ & GSTA5 & 2.7 & 3.3 & 1.5 & 2.9 & 2.9 & 1.0 \\
\hline 8136849 & $\begin{array}{l}\text { glutathione S- } \\
\text { transferase } \kappa 1\end{array}$ & GSTK1 & 5.7 & 5.5 & 0.8 & 7.3 & 7.8 & 1.5 \\
\hline 7903765 & $\begin{array}{l}\text { glutathione S- } \\
\text { transferase M1 }\end{array}$ & GSTM1 & 5.4 & 5.9 & 1.4 & 5.4 & 6.4 & 2.0 \\
\hline 8085370 & $\begin{array}{l}\text { glutathione S- } \\
\text { transferase M1-like }\end{array}$ & GSTM1L & 6.9 & 7.8 & 1.9 & 7.1 & 7.0 & 0.9 \\
\hline 7903753 & $\begin{array}{c}\text { glutathione S- } \\
\text { transferase M2 } \\
\text { (muscle) }\end{array}$ & GSTM2 & 4.5 & 6.3 & 3.5 & 5.3 & 5.4 & 1.1 \\
\hline 7918379 & $\begin{array}{c}\text { glutathione S- } \\
\text { transferase M3 } \\
\text { (brain) }\end{array}$ & GSTM3 & 6.2 & 6.2 & 1.0 & 6.1 & 6.4 & 1.3 \\
\hline 7903742 & $\begin{array}{l}\text { glutathione S- } \\
\text { transferase M4 }\end{array}$ & GSTM4 & 6.4 & 6.8 & 1.3 & 5.6 & 6.4 & 1.8 \\
\hline 7903777 & $\begin{array}{l}\text { glutathione S- } \\
\text { transferase M5 }\end{array}$ & GSTM5 & 4.1 & 6.1 & 4.0 & 4.3 & 4.1 & 0.9 \\
\hline 7930304 & $\begin{array}{l}\text { glutathione S- } \\
\text { transferase } \omega 1\end{array}$ & GSTO1 & 6.2 & 5.4 & 0.6 & 7.9 & 7.7 & 0.9 \\
\hline 7930311 & $\begin{array}{l}\text { glutathione S- } \\
\text { transferase } \omega 2\end{array}$ & GSTO2 & 5.2 & 5.8 & 1.5 & 6.8 & 7.1 & 1.2 \\
\hline 7941936 & $\begin{array}{l}\text { glutathione S- } \\
\text { transferase } \pi 1\end{array}$ & GSTP1 & 9.1 & 8.8 & 0.8 & 11.6 & 11.6 & 1.0 \\
\hline 8074980 & $\begin{array}{l}\text { glutathione S- } \\
\text { transferase } \theta 1\end{array}$ & GSTT1 & 5.2 & 4.8 & 0.8 & 7.1 & 7.1 & 1.0 \\
\hline 8071809 & $\begin{array}{l}\text { glutathione S- } \\
\text { transferase } \theta 2\end{array}$ & GSTT2 & 7.5 & 8.0 & 1.5 & 6.4 & 5.9 & 0.7 \\
\hline 8074972 & $\begin{array}{l}\text { glutathione S- } \\
\text { transferase } \theta \\
\text { pseudogene } 1\end{array}$ & GSTTP1 & 3.6 & 4.4 & 1.7 & 4.0 & 3.8 & 0.9 \\
\hline 7954196 & $\begin{array}{c}\text { microsomal } \\
\text { glutathione S- } \\
\text { transferase } 1\end{array}$ & MGST1 & 5.9 & 4.8 & 0.5 & 3.3 & 4.1 & 1.8 \\
\hline 8097513 & $\begin{array}{c}\text { microsomal } \\
\text { glutathione S- } \\
\text { transferase } 2\end{array}$ & MGST2 & 7.0 & 5.9 & 0.5 & 7.4 & 7.4 & 1.0 \\
\hline 7906978 & $\begin{array}{c}\text { microsomal } \\
\text { glutathione S- } \\
\text { transferase } 3\end{array}$ & MGST3 & 7.9 & 7.3 & 0.7 & 8.4 & 8.8 & 1.3 \\
\hline
\end{tabular}


Table II. Regulation of gene expression and ingenuity analysis of the 10 most common pathways involved in response of RMS to vincristine. ${ }^{\mathrm{a}}$

ID Molecules in network $\quad$ Score $\begin{aligned} & \text { Focus Top functions } \\ & \text { molec. }\end{aligned}$

1 ACR, ADCY, ADORA3, ADORA2B, AVPR1B, BMP15, C20ORF70, CALCA, CHI3L1, CHRM1, CHRM5, DRD5, ELF5, ERK, ETS, FGF9, FGF23, FSHR, G ai, GPR182, Gs-coupled receptor, HTR1A, HTR1D, KCNA2, KIR2DS2 (includes EG:3807), LHCGR, LTB, P2RY2, P2RY6, PLC, RAMP2, SP7, SPRED1, TDGF1, TNFAIP6

2 Ap1, C19ORF16, Cacna1, CACNA1C, Cacna1c/d/f/s, CACNA1D, CACNA1S, Creb, CTRC, CXCL1, FAM83C, GDF10, GLRX2, IGFBP4, KCNJ16, KLK2, KLK14, KLK1, (includes EG:3816), KLK5 (includes EG:25818), KRT16, L-type Calcium Channel, LBP, MYBPH, P38 MAPK, Pka, PRAP1, PRSS3 (includes EG:5646), PTGES, SERPIND1, SSTR2, TF, TFPI, TGM4, TXNDC2, VTN

3 ARHGAP29, CCL8, CCR7, CD2, CD8, CD8A, CD8B, CDH5, CEACAM1, CEACAM8, CHRNA2, CHRNA3, CHRNA4, Ggt, GGTLC1, GGTLC2, Ifn $\gamma$, Il12 p70, IL1F5, MEOX1, MT3, Nfat, NFATC2, NFkB, Nicotinic acetylcholine receptor, PAEP, PTPRCAP, Rac, Ras homolog, REG3G, RHOH, RND2, TCR, TLR10, ZNF675

4 AICDA, Akt, ALDH3A1, CD37, CD69, CD209, CD1D, CRP, GRIN2B, HLA-DQA1, HPSE, IFIT1L, Ifn $\alpha$, Ige, IGH, IGHA1, IGHM, Igm, IL1, IL12, IL18, IL19, IL24, Interferon $\alpha$, MHC Class II, Nos, NTF3, PIGR, PTPRH, PYHIN1 (includes EG:149628), STAT, STAT5a/b, TCL1A, USP8, VPREB3

5 AIRE, $\underline{\text { ARID4A }}$, ATP13A5, ATPase, BRCA2, Caspase, CHAT (includes EG:1103), COL9A1, CRABP1, CYP4F2, DQX1, E2f, FLT3, GAL, GDF2, GH1, Glutathione transferase, GST, GSTA1, GSTA5, GSTM2, GSTM5, HR, LEFTY2, Mapk, Mek, MST1, PRLH, RAD51, Ras, RBL1, Rxr, Thyroid hormone receptor, TRIP11, Vegf

6 ABLIM3, Adaptor protein 2, ALP, ALPP, ASCC3, ASGR2, BDKRB2, Calmodulin, Calpain, CASP12 (includes EG:120329), CES1 (includes EG:1066), CHRDL2, CORIN, F Actin, GABRA6, GABRB1, Hsp70, HSPA6, IQCB1, Jnk, LDL, NCR3, NPPA, OPN1LW (includes EG:5956), Opsin, Pkc(s), Pld, PRKCB1, RHO, SAG, SYT5, SYT6, SYT7, Tgf ß, TWIST1

7 ANK2, ATN1, ATXN1, CCDC116, CDH1, CLEC4F, CRYAB, CRYBA1, EWSR1, GLYATL1, GPRIN2, HDHD3, KRTAP3-2, KRTAP4-12, MDFI (includes EG:4188), MEGF11, MGC42630, Mmp, MMP28, MORC4, OSTALPHA, RAB37, RPS28, RXRA, SKIL, SSPO, TCEB3B, TINAGL1, TNF, TRIP13, UIMC1, WNK2, ZCCHC13, ZNF439, ZNF440
45

29

29

42

36
Cell signaling, molecular transport, nucleic acid metabolism

Cardiovascular disease, organismal injury and abnormalities, protein synthesis

Immune response, cardiovascular disease, ophthalmic disease

Immunological disease, cell-to-cell signaling and interaction, immune response

Cellular assembly and organization, DNA replication, recombination, and repair, nutritional disease

Cell signaling, nervous system development and function, visual system development and function

Cellular compromise, hepatic system disease, cell morphology 
Table II. Continued.

ID Molecules in network

Score

Focus Top functions

molec.

8 AMPD1, AQP7, ARL2, BCAS3, CABP5, CALD1, CaMKII, 20 Ck2, Cyclin A, Cyclin E, DMD, GDA, HCK, Histone h3, HOXA11, IL3, Insulin, LPO, MSTN, Myosin, NEFH, NPDC1, PDGF BB, PI3K, PLB1, PPP2R2B, PRG2 (includes EG:5553), RNA polymerase II, SLC5A2, SNTG1, TFF1, TRPM7, Tubulin, WFIKKN2, WWC1

9 APP, B4GALNT1, BHLHB2, CD207, CD209, CDH11, CHAT (includes EG:1103), COL4A6, COL6A2, COL8A1, COMP, CTCF, FBXW10, FCGR3A, GAL, HOXB5, HOXD3, IGHM, IL4, ITGA1, KIF5A, KIF5C, KIRREL2, KLC1, KLC2, LSP1, MATN1, MGC29506, PIGR, progesterone, TACSTD2, TGFB1, TJP1, TSPAN7, TUBA3E

10 ABCC6, ACCS, AKR1B1, BAZ1B, C6ORF123, C6ORF208, CCND1, CEBPA, CRYZL1, DTWD1, EPB41L4B, F7, FBL, FEZF2, GAPDH, GH1, HNF4A, HPN, IL15, MAP3K3, MDM2 (includes EG:4193), MYO1A, NME1, NPAS4, PROZ (includes EG:8858), RXRA, SERPINA10, SERPINE1, SMAD3, SMARCA5, STRAP, TRAF6, TTC25, VHL, ZNF253
17

Genetic disorder, skeletal and muscular disorders, cell-to-cell signaling and interaction

Cellular movement, hematological system development and function, immune response

Cancer, cellular growth and proliferation, gene expression

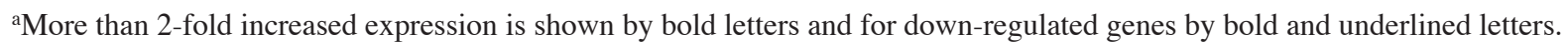

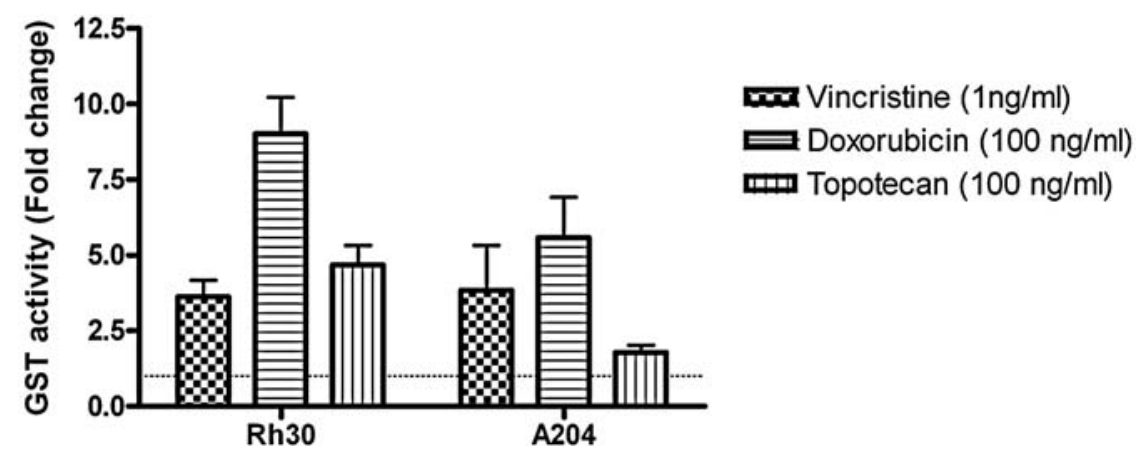

Figure 3. GST activity after treatment with cytotoxic agents for $72 \mathrm{~h}$ in alveolar (Rh30) and embryonal (A204) rhabdomyosarcoma cell lines. Induction of the GST activity was assumed if the fold change was greater than control experiments which were set as 1 (dotted line).

likelihood that the assembly of a set of focus genes in a network has at least a high confidence of not being generated by random chance alone. The number of molecules changed in the network based on the differential analysis is given as focus molecules. Top functions involved cell signaling, replication and immune response.

Modulation of GST activity by cytotoxic agents. To determine whether vincristine-induced changes in GST mRNA were mirrored by altered protein expression, we performed an analysis of GST activity with the human RMS cell lines Rh30 and A204. As GST is involved in detoxification of different cytotoxic agents, we included topotecan and doxorubicin in the analysis, which are also used in the treatment of childhood RMS. Incubation with cytotoxic agents for $72 \mathrm{~h}$ led to an induction of specific GST activity in alveolar RMS (Rh30) after treatment with vincristine [Fold change (FC): 3.6 \pm 0.95 ], with doxorubicin (FC: $9 \pm 2.09$ ) and with topotecan (FC: $4.7 \pm 1.15$ ). In embryonal RMS (A204), induction was observed after treatment with vincristine (FC: $3.8 \pm 2.6$ ), doxorubicin (FC: $5.6 \pm 2.3$ ) and topotecan (FC: $1.8 \pm 0.42$, Fig. 3).

After incubation with cytotoxic agents for $144 \mathrm{~h}$, we found an induction of the GST activity in alveolar RMS after treatment with vincristine (FC: $2.4 \pm 0.13$ ). Enhancement of the GST activity was found after treatment with doxorubicin 


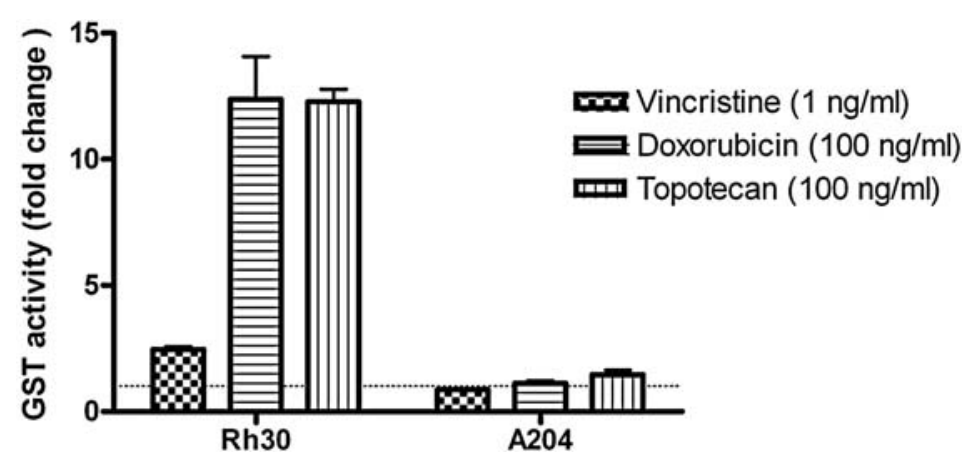

Figure 4. GST activity after incubation with cytotoxic agents for $144 \mathrm{~h}$ in alveolar (Rh30) and embryonal (A204) RMS cells. Changes in GST activity were related to untreated cultures, which are depicted as a dotted line.

(FC: $12.4 \pm 1.7$ ) and topotecan (FC: $12.3 \pm 0.48)$ compared to the experiments with an incubation time of $72 \mathrm{~h}$. Highest induction of GST activity was found in Rh30 cells after treatment with doxorubicin. No induction of the GST activity was found in A204 cells (Fig. 4).

Modulation of anticancer drug activity with GST inhibitors. In order to investigate a possible inhibition of GST, we studied the GST inhibitors etacrinic acid and the recently described OZO-H (4-phenyl-1,3,2-oxathiazolyium-5-oleate) in a proliferation assay. RMS cell viability in cultures treated with GST inhibitors decreased significantly depending on the type of tumor cells and inhibitor used. In cultures of alveolar RMS cells, etacrinic acid was most effective, but did not inhibit viability $>50 \%$ at tested concentrations (Fig. 5A). Embryonal RMS cells were growth inhibited by OZO-H with an $\mathrm{IC}_{50}$ of $8 \pm 1.7 \mu \mathrm{M}$. Etacrinic acid was less effective in embryonal RMS cells and showed comparable growth inhibition as in alveolar RMS cells (Fig. 5A).

A combination of GST inhibitors and cytotoxic agents may modulate drug sensitivity of tumor cells. The effects of cytotoxic agents on tumor cell viability in cells treated with GST inhibitors were tumor cell type-dependent. We found a significant additive effect on cell death in alveolar RMS cells after treatment with vincristine, doxorubicin and topotecan combined with different concentrations of the GST inhibitors (Fig. 5B). The additive effect on cell death was observed in embryonal RMS only for combination of GST inhibitors with doxorubicin and topotecan (Fig. 5C).

\section{Discussion}

Chemotherapy plays an essential role in the treatment of RMS regarding the reduction of the initial tumor mass as well as control of circulating tumor cells in order to avoid metastatic invasion (17). After several cycles of chemotherapy multidrug resistance (MDR) may develop and reduces the effectiveness of the cytotoxic agents (2). We previously described that MDR plays a role in childhood RMS by known mechanisms such as P-glycoprotein in alveolar subtype and multidrug resistance-associated protein (MRP) and lung resistance-related protein (LRP) in embryonal subtype (10). Although these mechanisms might be responsible for MDR in rhabdomyosarcoma in part, a complete reversal of MDR could not be achieved by MDR inhibitors (18). Therefore, other mechanisms of MDR may be responsible in this tumor entity.

As a model to assess MDR, drug-resistant cells may be selected in vitro by increasing doses of drugs in cell cultures (19). In our hands, the used RMS cell lines could not be maintained for longer than 2 weeks in culture with vincristine at concentrations higher than $\mathrm{IC}_{50}$. Therefore, we employed an animal model of xenotransplanted RMS treated with vincristine. The effectiveness of chemotherapy with vincristine in RMS has been demonstrated previously by our group (10). Despite the long treatment of the tumors, we detected vital tumor cells after chemotherapy, indicating a selection of multidrug-resistant tumor cells in this model. Oligonucleotide microarray analysis identified more than 1000 genes regulated after vincristine treatment, suggesting a profound change of the phenotype of the RMS cell. Besides several genes regulating ATP transporters, we found genes regulating the GST family, possibly involved in multidrug resistance.

GSTs are enzymes that detoxify cytotoxic agents within the cancer cell contributing to chemotherapy resistance (20). GSTs are divided into two groups: the membrane-bound microsomal and cytosolic family members (11). Cytosolic GSTs are divided into six classes $(\alpha, \mu, \omega, \pi, \theta, \xi)(11)$. GST expression pattern influences cancer susceptibility, prognosis and treatment (21). Especially GST- $\pi$ plays an important role in mediating resistance to cytotoxic agents (21). High levels of GSTs have been found in a variety of tumors (12). GSTmediated MDR has been reported in breast cancer (20), ovarian carcinomas (22), head and neck cancer (23) as well as lung squamous-cell carcinoma (24). In human RMS tissue samples GST expression was observed using microarray mRNA analysis. Among different isotypes of GST, GST- $\pi$ was expressed at high levels comparable with normal muscle tissue whereas GSTA2 was detected preferentially in RMS tissue when compared with Ewing sarcoma (GDS971 and GDS1562 on NCBI, GEO Profiles). In our model GSTA2 was induced in Rh30 tumors, whereas the GST- $\mu$ enzyme family was up-regulated by vincristine in Rh30 and A204 tumors. A positive correlation between GST- $\pi$ expression and resistance of soft tissue sarcoma on adriamycin, cisplatin and mitomycin $\mathrm{C}$ was emphasized (11).

Second line drugs used in the systemic therapy in relapsed RMS vincristine, doxorubicin and topotecan induced GST 

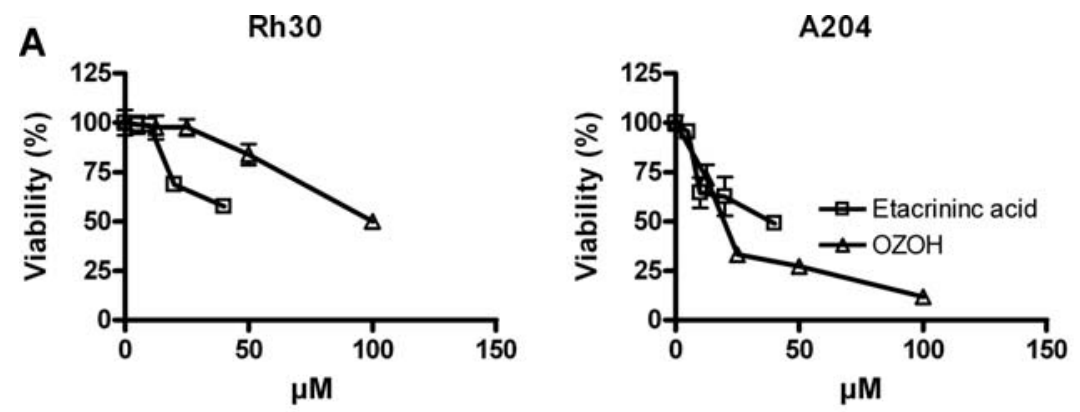

B

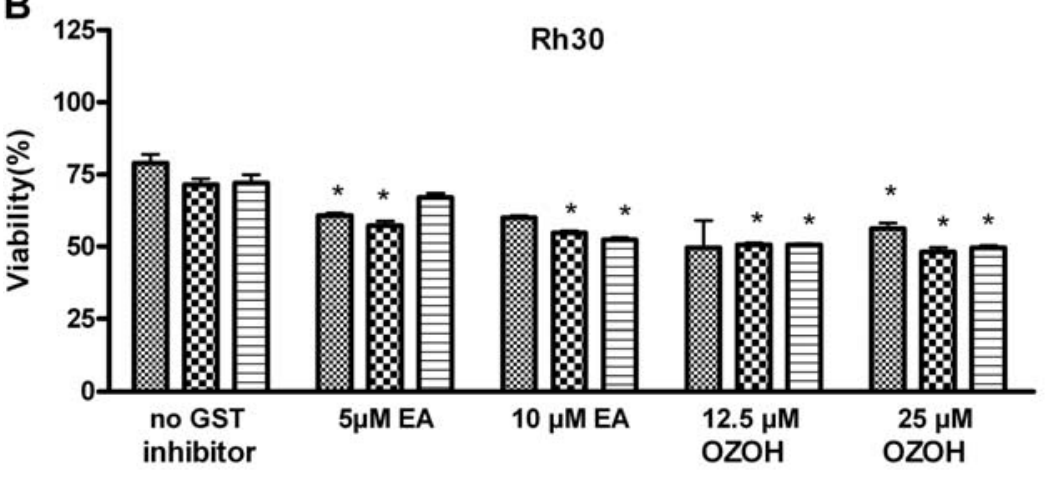

C

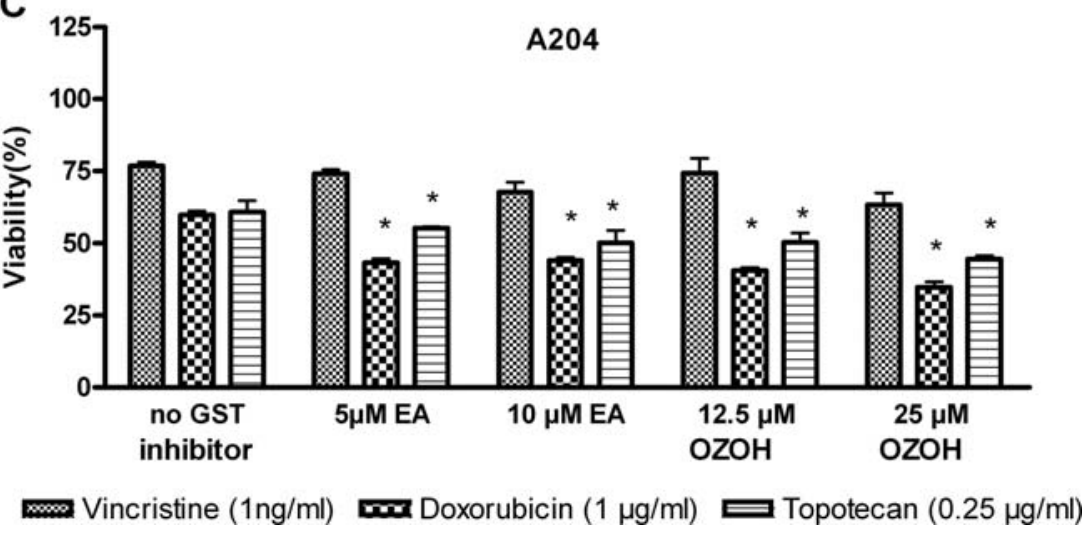

Figure 5. Effects of the GST inhibitor EA and OZO-H on tumor cell viability in RMS cells. (A) Rh30 and A204 cells were treated with increased concentrations of GST inhibitors EA and OZOH. Viability was measured in a proliferation assay $72 \mathrm{~h}$ later. Combination of GST inhibitor with vincristine, doxorubicin and topotecan revealed additive effects for Rh30 cells (B) and A204 cells (C). A significant reduction of tumor cell viability could be shown using the GST inhibitors in these cell cultures compared with control experiments without GST inhibitors ("ANOVA rank test $\mathrm{p}<0.05$ ).

activity in our RMS-cell cultures. These denote a general reaction of RMS on cytotoxic drugs with increased expression of detoxification enzymes like GST. GST activity depended on the tumor cell line as well as the cytotoxic agent used. Treatment with vincristine had an early effect on the GST activity in all cell lines suggesting that GST activation after vincristine treatment is an acute effect. This is of clinical importance as vincristine is only applied at days 1 and 2 within one treatment cycle in the treatment protocol of the Cooperative Soft Tissue Sarcoma Study CWS 2002-P of the German Association of Pediatric Hematology and Oncology (6). After treatment with doxorubicin and topotecan similar observations were found in embryonal RMS. In contrast, in alveolar RMS an increase in the GST activity was found after longer incubation times with doxorubicin and topotecan. These findings seem to indicate that GST activity depends on the tumor cell type. This might be caused through an altered capacity to regulate kinase-dependent proliferation pathways (11). GSTs interact with critical kinases involved in apoptosis and proliferation. Tumor cells with enhanced expression of GSTs can sequestrate kinases like JNK and ASK1 inhibiting the sustained activation of downstream kinases and the consequent apoptosis induction $(11,25)$.

The expression pattern in RMS and the multiple functionalities of GSTs in detoxification, in redox balance and in kinase inhibition renders GSTs to be attractive targets to improve chemotherapy of RMS. Up to now, the effects of GST inhibitors have not been studied in human RMS cells. We used the common GST inhibitor etacrininc acid and the novel GST inhibitor OZO-H, which was initially described by Cui et al (25). Application of OZO-H leads to a dissociation of the GST- $\pi-J N K$ complex activating the JNK/ Jun pathway in leukemia cells (25). Treatment with GST inhibitors led to a reduction of tumor cell viability depending on the RMS cell line used. A204 cells showed the highest expression of GST- $\pi$ in microarray analysis and respond better to OZO-H than Rh30 cells. Up to now, an effect of OZO-H has only been described in leukemia and breast cancer cells 
(25). It remains unclear whether OZO-H is effective in broad set of tumor entities. Etacrininc acid binds to and inhibits GST, however, it depletes the glutathione pool by conjugation and may induce cell death by altering redox potential of the cell (26). Therefore, the observed cell death in RMS cell cultures with etacrinic acid may be a result of DNA damage additional to GST inhibition. Other inhibitors of GSTs such as TLK199 or TLK286 given as prodrugs are activated by GST itself and can alkylate cellular nucleophiles and downregulate GST- $\pi$ expression (27). These drugs are presently tested in Phase III settings for non-small cell lung and ovarian cancers (28).

Inhibition of GSTs has been previously described to modulate drug resistance by sensitization of tumor cells to cytotoxic agents (11). Rat-derived RMS cell lines respond to inhibition of glutathione synthesis with enhanced sensitivity to vincristine depending on the cell line used (29). In human RMS cells GST inhibitors had a moderate additive effect to cytotoxic drugs on cell death. As the drug-induced GST activity was higher in Rh30 cells than in A204 cells we expected a parallel drop of cell viability when cultured together with GST inhibitors. However, A204 cells responded better to a combination treatment which may be associated with the higher level of GST- $\pi$ expression. As vincristine induced preferentially the GST- $\mu$-specific, inhibitors of this enzyme family may be more effective as combination therapy in RMS cells.

In conclusion, GSTs seems to play a role for chemotherapy resistance in childhood RMS in vitro and in vivo in different histological subtypes. Inhibition of GSTs by GST inhibitors as strategy for a possible reversal of MDR is feasible, but tumor cell-dependent. New GST inhibitors have to be developed as targeting specific GST isoforms, which are upregulated in RMS.

\section{References}

1. Fuchs J, Wenderoth M, von Schweinitz D, Haindl J and Leuschner I: Comparative activity of cisplatin, ifosfamide, doxorubicin, carboplatin, and etoposide in heterotransplantated hepatoblastoma. Cancer 83: 2400-2407, 1998.

2. Cocker HA, Pinkterton CR and Kelland LR: Characterization and modulation of drug resistance of human rhabdomyosarcoma cell lines. Br J Cancer 83: 338-345, 2000.

3. Crist WM, Anderson JR, Meza JL, et al: Intergroup rhabdomyosarcoma study-IV: results of patients with non-metastatic disease. J Clin Oncol 19: 3091-3102, 2001.

4. Newton WA Jr, Gehan EA, Webber BL, et al: Classification of rhabdomyosarcomas and related sarcomas. Cancer 76: 1073-1085, 1995.

5. Lawrence W Jr, Anderson JR, Gehan EA and Maurer H: Pretreatment TNM staging of childhood rhabdomyosarcoma: a report of the Intergroup study group. Children's cancer study group. Pediatric Oncology Group. Cancer 80: 1165-1170, 1997.

6. Treuner J and Koscielniak E: Cooperative Weichteilsarkom Studie CWS 2002-P. Study protocol, 2003.

7. Stevens MC, Rey A, Bouvet N, et al: Treatment of nonmetastatic rhabdomyosarcoma in childhood and adolescence: third study of the international society of paediatric oncology SIOP malignant mesenchymal tumor 89. J Clin Oncol 23: 2586-2587, 2005.

8. Meza JL, Anderson J, Pappo AS, Meyer WH and Children's Oncology Group: Analysis of prognostic factors in patients with nonmetastatic rhabdomyosarcoma treated on intergroup rhabdomyosarcoma studies III and IV: the children's oncology group. J Clin Oncol 24: 3844-3851, 2006.

9. Ferrari A, Casanova M, Bisogno G, et al: Rhabdomyosarcoma in infants younger than one year old: a report from the Italian Cooperative Group. Cancer 97: 2597-2604, 2003.

10. Seitz G, Warmann SW, Vokuhl CO, et al: Effects of standard chemotherapy on tumor growth and regulation of multidrug resistance genes and proteins in childhood rhabdomyosarcoma. Pediatr Surg Int 23: 431-439, 2007.

11. Townsend DM and Tew KD: The role of glutathione-Stransferase in anti-cancer drug resistance. Oncogene 22: 73697375, 2003.

12. Tew KD: Glutathione-associated enzymes in anticancer drug resistance. Cancer Res 54: 4313-4320, 1994.

13. Depeille P, Cuq P, Mary S, Passagne I, Evrard A, Cupissol D and Vian L: Glutathione S-transferase M1 and multidrug resistance protein 1 act in synergy to protect melanoma cells from vincristine effects. Mol Pharmacol 65: 897-905, 2004.

14. Tew KD, Bomber AM and Hoffman SJ: Ethacrynic acid and piriprost as enhancers of cytotoxicity in drug resistant and sensitive cell lines. Cancer Res 48: 3622-3625, 1988.

15. Wu Z, Irizarry RA, Gentleman R, Murillo FM and Spencer F: A model based background adjustment for oligonucleotide expression arrays. Department of Biostatics Working Papers, John Hopkins University, Baltimore, MD, 2003.

16. Benjamini Y and Hochberg Y: Controlling the False Discovery Rate: a practical and powerful approach to multiple testing: Journal of the Royal Statistical Society B 57: 289-300, 1995.

17. Singer D, Demetri GD, Baldini EH and Fletcher CD: Management of soft-tissue sarcoma: an overview and update. Lancet Oncol 1: 75-85, 2000.

18. Cowie FJ, Pinkterton CR, Phillips M, Dick M, Judson I, McCarthy PT and Flanagan RJ: Continuous-infusion verapamil with etoposide in relapsed or resistant paediatric cancers. Br J Cancer 71: 877-881, 1995.

19. Kotchetkov R, Cinatl J, Blaheta R, et al: Development of resistance to vincristine and doxorubicine in neuroblastoma alters malignant properties and induces additional karyotype changes: a preclinical model. Int J Cancer 104: 36-43, 2003.

20. Su F, Hu X, Jia W, Gong C, Song E and Hamar P: Gluthation S Transferase $\pi$ indicates chemotherapy resistance in breast cancer. J Surg Res 113: 102-108, 2003.

21. McIlwain CC, Townsend DM and Tew KD: Glutathione Stransferase polymorphisms: cancer incidence and therapy. Oncogene 25: 1639-1648, 2006.

22. Mayr D, Pannekamp U, Baretton GB, et al: Immunohistochemical analysis of drug resistance-associated proteins in ovarian carcinomas. Pathol Res Prac 196: 469, 2000.

23. Shiga H, Heath EI, Rasmussen AA, et al: Prognostic value of p53, glutathione S-transferase pi, and thymidylate synthase for neoadjuvant cisplatin-based chemotherapy in head and neck cancer. Clin Cancer Res 5: 4097-4104, 1999.

24. Inoue T, Ishida T, Sugio K, Maehara Y and Sugimachi K: Glutathione $\mathrm{S}$ transferase $\mathrm{Pi}$ is a powerful indicator in chemotherapy of human lung squamous-cell carcinoma. Respiration 62: 223-227, 1995.

25. Cui H, Shen J, Lu D, Zhang T, Zhang W, Sun D and Wang PG: 4-Aryl-1,3,2-oxathiazolylium-5-olate: a novel GST inhibitor to release JNK and activate c-Jun for cancer therapy. Cancer Chemother Pharmacol 62: 509-515, 2008.

26. Ploemen JH, Van Schanke A, Van Ommen B and Van Bladeren PJ: Reversible conjugation of ethacrynic acid with glutathione and human glutathione S-transferase P1-1. Cancer Res 54: 915-919, 1994.

27. Rosario LA, O'Brien ML, Henderson CJ, Wolf CR and Tew KD: Cellular response to a gluthatione S-transferase P1-1 activated prodrug. Mol Pharmacol 58: 167-174, 2000.

28. Tew KD: TLK-286: a novel glutathione S-transferase-activated prodrug. Expert Opin Investig Drugs 14: 1047-1054, 2005.

29. Castro B, Alonso-Varona A, del Olmo M, Bilbao P and Palomares T: Role of gamma-glutamyltranspeptidase on the reponse of poorly and moderately differentiated rhabdomyosarcoma cell lines to buthionine sulfoximine-induced inhibition of glutathione synthesis. Anticancer Drugs 13: 281-291, 2002. 